\title{
Public Attitudes towards Cancer Survivors among Korean Adults
}

\section{Su Yeon Kye, $\mathrm{PhD}$ \\ Hyun Jeong Lee, MD \\ Yeonseung Lee, MSW \\ Young Ae Kim, PhD}

Division of Cancer Control and Policy, National Cancer Control Institute, National Cancer Center, Goyang, Korea
Correspondence: Young Ae Kim, PhD Division of Cancer Control and Policy, National Cancer Center, 323 Ilsan-ro,

Ilsandong-gu, Goyang 10408, Korea

Tel: 82-31-920-2947

Fax: 82-31-920-2169

E-mail: elkim7@gmail.com

Received May 15, 2019

Accepted February 5, 2020

Published Online February 6, 2020

\section{Purpose}

We evaluated public attitudes towards cancer survivors and identified the characteristics associated with these attitudes in Korea.

\section{Materials and Methods}

We performed this cross-sectional study using proportionate quota random sampling of the 2015 Korean Census. In May 2017, investigators conducted face-to-face interviews with 1,500 Korean volunteers aged between 20 and 79 years. The questionnaire recorded sociodemographic factors, smoking and drinking habits, cancer history in family and acquaintances, interest in cancer survivors, cancer-survivor blame, and attitudes towards cancer survivors.

\section{Results}

Many participants had negative attitudes towards cancer survivors. People with a monthly household income above US $\$ 7,000$ were less likely to have a negative attitude than those with monthly incomes below US $\$ 1,499$. People in their 70 s, without a religion, living in rural areas, smokers, or those who blame cancer survivors for their own cancer were more likely to have a negative attitude than people outside these categories. People interested in cancer survivors were less likely to have a negative attitude than those who were not interested.

\section{Conclusion}

To improve attitudes towards cancer survivors, it will be necessary to increase interest in cancer survivors through education, publicity, and advocacy using strategic messaging that focuses on social and institutional aspects and emphasizes that responsibility for cancer should not be attributed to cancer patients. Inducing the public to be interested in cancer survivors will be important for positive attitudes toward cancer survivors.

\section{Introduction}

There were an estimated 14 million patients a year worldwide with cancer in 2012, and cancer incidence is projected to increase by $50 \%$ to 21.6 million patients a year by 2030 $[1,2]$. In parallel, the number of cancer survivors has also increased and is expected to further increase with more frequent early diagnosis, improved treatment, and an aging population.

\section{Key words}

Public, Attitude, Cancer survivor, Korean 
An increasing number of cancer prevention campaigns supported by well-established theories about healthy behaviors have improved public health communication, leading to increased public awareness about the lifestyle risk factors related to cancer [5]. However, these campaigns also resulted in a tendency towards a negative public response to individuals with modifiable conditions; moreover, survivors of controllable cancers became more likely to blame themselves for their illness [5,6].

Cancer survivors' perception of stigma, blame, and other negative reactions can lead to self-blame, shame, and fear of discrimination. In turn, these can cause medical and psychosocial problems in cancer survivors and contribute to insufficient patient-clinician communication, distress, depression, physical and emotional maladjustment, reduced treatment adherence, and a poor quality of life [7-9].

In Korea, the 5-year relative cancer survival rate has increased remarkably from $41.2 \%$ in $1993-1995$ to $70.7 \%$ in 2011 2015, with 1.61 million cancer survivors in 2015 [10]. However, despite increased cancer survivorship resulting from highly developed medical technology, the social environment for cancer survivors remains insufficient. For example, a large proportion of patients were less willing to disclose their cancer diagnosis even to family members, and one-third of cancer survivors had negative attitudes towards cancer and stereotypical views about themselves [11,12]. A nationwide-cohort study showed that $47 \%$ of cancer survivors lost their jobs after receiving their cancer diagnosis and only $30.5 \%$ were re-employed [13]. Moreover, the frequency of suicide attempts is three times higher among cancer survivors than the general public [14].

The problems of cancer survivors are probably worsened by negative perceptions and prejudices formed by the general public about cancer survivors. Therefore, our objective in this study was to evaluate public attitudes towards cancer survivors and identify the characteristics associated with these attitudes in Korea.

\section{Materials and Methods}

\section{Study design and population}

Our goal was to survey 1,500 members of the general public in Korea who were between 20-79 years old and had no immediate personal experience of cancer. Initially, we stratified samples by age and sex for each of the 17 administrative districts (cities and provinces) based on the 2015 Korean Census, then obtained the sample size using a probability proportional to size method. Of the randomly selected 2,190 responders who were telephoned, we excluded 690 responders from the interview due to absence, refusal to participate for various reasons, or because the responder was a cancer patient. Finally, a total of 1,500 participants were interviewed (response rate, 68.5\%), and face-to-face interviews were conducted by professional interviewers from the Metrix Corporation (Seoul, Korea) in May 2017. Our researchers carefully reviewed and monitored the interview process.

\section{Measurements}

We used eight questions developed by Cho et al. [11] to assess attitudes towards cancer survivors. Details of tool development are described previously [11]. Question topics included the recognition of cancer survivors, sexual intimacy with cancer survivors, deserved protection in society for cancer survivors, contributions of cancer survivors to society, uncomfortable feelings associated with cancer survivors, avoidance of interaction with neighbors with cancer, avoidance of marriage to people with cancer-survivor family members, and avoidance of working with cancer survivors. These items were measured using a 4-point Likert scale ranging from 1 (strongly disagree) to 4 (strongly agree). We considered participants to have a negative attitude if their average score was over 3 point (covering possibly agree and strongly agree). Cronbach's alpha for these items was 0.76 .

Interest in cancer survivors was assessed by participants' response to the question "How much interest do you generally have in the cancer survivor problem?" This item was measured using a 5-point Likert scale ranging from 1 (not interested at all) to 5 (very interested). We combined "not interested at all" with "almost not interested" responses to form a "lowly interested" category and combined "very interested" with "mostly interested" to form a "highly interested" category. Cancer-survivor blame was measured by participants' response to the yes/no question "Are cancer survivors responsible for their cancer?" This item was developed from previous studies [6,7,15].

We also considered other variables, including sex, age, education, monthly household income, marital status, religion, employment, residential area, smoking and drinking habits, as well as cancer history of family and acquaintances. Education level was classified into three categories: less than middle school education, high school graduate, and college graduate or higher. Monthly household income was classified into four categories: $\leq \$ 1,499, \$ 1,500-\$ 3,999, \$ 4,000$ $\$ 6,999$, and $\geq \$ 7,000$. These cut-off points are the quartiles of monthly household income in Korea [16]. We divided participants into three occupational groups. White-collar workers were defined as professionals or educated workers who performed administrative or sales-coordination tasks. Bluecollar workers were defined as workers in industry or those 
who did physical labor. Residential areas were classified according to the size of the city. Seoul is the largest city in Korea with a population over 1,000,000. Areas classified as "metropolitan" included suburban areas of Seoul and the top six largest cities in Korea after Seoul with populations ranging from 100,000-300,000. "Rural" areas were small cities or counties with a total population $<100,000$.

\section{Statistical analysis}

We used descriptive statistics to summarize attitudes towards cancer survivors. A multivariate logistic regression analysis determined the adjusted odds ratios (aORs) for relationships between a negative attitude towards cancer survivors and sex, age, education, monthly household income, marital status, religion, occupation, residential area, smoking, drinking, cancer history in family or acquaintances, interest in cancer survivors, and cancer survivor blame. We performed all analyses using SAS ver. 9.4 (SAS Institute Inc., Cary, NC).

\section{Ethical statement}

We obtained written consent from all respondents after informing them that completion of the survey was voluntary, anonymous, and confidential. The study protocol was approved by the Institute Review Board at the National Cancer Center (NCC2017-0109). All procedures performed in studies involving human participants were in accordance with the ethical standards of the institutional and / or national research committee and with the 1964 Helsinki declaration and its later amendments or comparable ethical standards.

\section{Results}

In this study, $50.3 \%$ of participants were male, $12.4 \%$ of participants had less than a middle school education, 38.7\% had a high school education, and $48.9 \%$ had a college education or higher. For monthly household income, $17.7 \%$ of participants earned below US $\$ 1,499$ and 7.0\% earned above US $\$ 7,000$. We found that $72.1 \%$ of participants were married people, $5.3 \%$ were divorced / separated / widowed, and $22.6 \%$ were single; $46.3 \%$ of participants had a religion; $29.3 \%$ of participants were white-collar workers; $20.0 \%$ of participants lived in the capital city, and $54.5 \%$ lived in rural regions. Smokers were $27.2 \%$ and drinkers were $76.5 \%$ of participants. There was history of cancer for family or acquaintances in $54.5 \%$ of participants. The proportion of people who were highly interested in cancer survivors was $40.8 \%$, and
Table 1. Characteristics of study participants

\begin{tabular}{|c|c|}
\hline Variable & $\begin{array}{r}\text { No. }(\%) \\
(\mathrm{n}=1,500)\end{array}$ \\
\hline \multicolumn{2}{|l|}{ Sex } \\
\hline Male & $754(50.3)$ \\
\hline Female & $746(49.7)$ \\
\hline \multicolumn{2}{|l|}{ Age group (yr) } \\
\hline $20-29$ & $253(16.9)$ \\
\hline $30-39$ & $280(18.7)$ \\
\hline $40-49$ & $328(21.9)$ \\
\hline $50-59$ & $317(21.1)$ \\
\hline $60-69$ & $201(13.4)$ \\
\hline $70-79$ & $121(8.1)$ \\
\hline \multicolumn{2}{|l|}{ Education } \\
\hline Middle school graduate or less & $186(12.4)$ \\
\hline High school graduate & $580(38.7)$ \\
\hline College graduate or more & $734(48.9)$ \\
\hline \multicolumn{2}{|l|}{ Monthly household income (\$) } \\
\hline$\leq 1,499$ & $266(17.7)$ \\
\hline $1,500-3,999$ & $646(43.1)$ \\
\hline $4,000-6,999$ & $483(32.2)$ \\
\hline$\geq 7,000$ & $105(7.0)$ \\
\hline \multicolumn{2}{|l|}{ Marital status } \\
\hline Married & $1,082(72.1)$ \\
\hline Divorced/Separated/Widowed & $79(5.3)$ \\
\hline Single & $339(22.6)$ \\
\hline \multicolumn{2}{|l|}{ Religion } \\
\hline Yes & $695(46.3)$ \\
\hline No & $805(53.7)$ \\
\hline \multicolumn{2}{|l|}{ Employment } \\
\hline White-collar & $439(29.3)$ \\
\hline Blue-collar & $586(39.1)$ \\
\hline Unemployed & $475(31.7)$ \\
\hline \multicolumn{2}{|l|}{ Residential area } \\
\hline Seoul (capital city) & $300(20.0)$ \\
\hline Metropolitan & $383(25.5)$ \\
\hline Rural & $817(54.5)$ \\
\hline \multicolumn{2}{|l|}{ Smoking } \\
\hline Yes & $408(27.2)$ \\
\hline No & $1,092(72.8)$ \\
\hline \multicolumn{2}{|l|}{ Drinking } \\
\hline Yes & $1,148(76.5)$ \\
\hline No & $352(23.5)$ \\
\hline \multicolumn{2}{|c|}{ Cancer history (family or acquaintance) } \\
\hline Yes & $818(54.5)$ \\
\hline No & $682(45.5)$ \\
\hline \multicolumn{2}{|l|}{ Interest in cancer survivors } \\
\hline Low & $339(22.6)$ \\
\hline Moderate & $549(36.6)$ \\
\hline High & $612(40.8)$ \\
\hline \multicolumn{2}{|l|}{ Cancer-survivor blame } \\
\hline Yes & $746(49.7)$ \\
\hline No & $754(50.3)$ \\
\hline
\end{tabular}


Table 2. Attitudes toward cancer survivors

\begin{tabular}{|c|c|c|c|c|c|}
\hline & \multicolumn{4}{|c|}{ No. $(\%)$} & \multirow{2}{*}{ Mean $\pm \mathrm{SE}^{\mathrm{a}}$} \\
\hline & Strongly disagree & Disagree & Agree & Strongly agree & \\
\hline $\begin{array}{l}\text { Cancer survivors are easily recognized } \\
\text { through their looks }\end{array}$ & $85(5.7)$ & $782(52.1)$ & $575(38.3)$ & $58(3.9)$ & $2.40 \pm 0.02$ \\
\hline $\begin{array}{l}\text { Cancer survivors would have a difficult time } \\
\text { having sexual intimacy }\end{array}$ & $79(5.3)$ & $645(43.0)$ & $658(43.9)$ & $118(7.9)$ & $2.54 \pm 0.02$ \\
\hline Cancer survivors deserve to be protected in society & $17(1.1)$ & $325(21.7)$ & $936(62.4)$ & $222(14.8)$ & $2.91 \pm 0.02$ \\
\hline $\begin{array}{l}\text { Cancer survivors would not be able to } \\
\text { make contributions to society }\end{array}$ & $176(11.7)$ & $980(65.3)$ & $323(21.5)$ & $21(1.4)$ & $2.12 \pm 0.02$ \\
\hline I feel uncomfortable when I am with cancer survivors & $186(12.4)$ & $707(47.1)$ & $524(34.9)$ & $83(5.5)$ & $2.34 \pm 0.02$ \\
\hline $\begin{array}{l}\text { I tend to avoid interacting with neighbors } \\
\text { who are cancer survivors }\end{array}$ & $188(12.5)$ & $890(59.3)$ & $371(24.7)$ & $51(3.4)$ & $2.19 \pm 0.02$ \\
\hline $\begin{array}{l}\text { I would avoid marrying people whose } \\
\text { family members are cancer survivors }\end{array}$ & $64(4.3)$ & 488 (32.5) & 775 (51.7) & 173 (11.5) & $2.70 \pm 0.02$ \\
\hline I would avoid working with cancer survivors & $182(12.1)$ & $854(56.9)$ & $420(28.0)$ & $44(2.9)$ & $2.22 \pm 0.02$ \\
\hline
\end{tabular}

$\mathrm{SE}$, standard error. ${ }^{\mathrm{a})} \mathrm{A}$ higher mean indicates a higher negative attitude to cancer survivors.

$49.7 \%$ of participants attributed some blame for cancer to cancer survivors (Table 1).

The proportion of participants who agreed or strongly agreed that cancer survivors are easily recognized by their looks was $42.2 \%$. We found that $51.8 \%$ agreed or strongly agreed that cancer survivors would have a difficult time having sexual intimacy, $77.2 \%$ agreed or strongly agreed that cancer survivors deserve to be protected in society, and $22.9 \%$ agreed or strongly agreed that cancer survivors would not be able to contribute to society. Additionally, $40.4 \%$ of participants agreed or strongly agreed that they felt uncomfortable when in the presence of cancer survivors, $28.1 \%$ agreed or strongly agreed that they tended to avoid interacting with neighbors who are cancer survivors, $63.2 \%$ agreed or strongly agreed that they would avoid marrying people with cancer-survivor family members, and $30.9 \%$ agreed or strongly agreed that they would avoid working with cancer survivors (Table 2).

Table 3 provides aORs for associations between negative attitudes towards cancer survivors and sociodemographic factors, smoking and drinking habits, cancer history of family and acquaintances, interest in cancer survivors, and cancer-survivor blame. People in their 70s were significantly more likely to have a negative attitude than their 20s (odds ratio [OR], 4.11; 95\% confidence interval [CI], 1.30 to 10.97). We found that a monthly household income above US \$7,000 was significantly associated with a lower likelihood of a negative attitude towards cancer survivors than an income below US \$1,499 (OR, 0.59; 95\% CI, 0.36 to 0.94). People who had no religion were significantly more likely to have a negative attitude than those who did (OR, 1.50; 95\% CI, 1.02 to
2.22). Rural residents were significantly more likely to have negative attitude towards cancer survivors than capital city residents (OR, 1.42; 95\% CI, 1.07 to 1.88). Nonsmokers were significantly less likely to have a negative attitude towards cancer survivors (OR, 0.58; 95\% CI, 0.35 to 0.95). Those who indicated interest in cancer survivors were less likely to have a negative attitude towards cancer survivors (moderate OR, 0.68; 95\% CI, 0.51 to 0.91; high OR, 0.36; 95\% CI, 0.22 to 0.60 ). Participants who attributed blame to cancer survivors were significantly more likely to have a negative attitude towards cancer survivors than those who did not (OR, 1.36; 95\% CI, 1.09 to 1.67$)$.

\section{Discussion}

This study evaluated public attitudes towards cancer survivors and identified the public attitude associations in the Korean population. Many participants had negative attitudes about cancer survivors, including stereotyped, discriminatory, and prejudiced views. People with a monthly household income above US $\$ 7,000$ were less likely to have a negative attitude than those with monthly incomes below US $\$ 1,499$. People in their 70 s, who had no a religion, who lived in rural areas, or smoked were more likely to have a negative attitude towards cancer survivors than those who did not fall into these categories. People who have interest in cancer survivors were less likely to have a negative attitude than those who were not interested. People who hold cancer survivors 
Table 3. Factors associated with negative attitude toward cancer survivors ${ }^{\text {a) }}$

Variable Percent

Sex

Male

Female

Age group (yr)

20-29

30-39

40-49

50-59

60-69

70-79

Education

Middle school graduate or less

High school graduate

College graduate or more

Monthly household income (\$)

$$
\leq 1,499
$$

$1,500-3,999$

4,000-6,999

$\geq 7,000$

Marital status

Married

Divorced/Separated/Widowed

Single

Religion

Yes

No

Employment

White-collar

Blue-collar

Unemployed

Residential area

Seoul (capital city)

Metropolitan

Rural

Smoking

Yes

No

Drinking

Yes

No

Cancer history (family or acquaintance)

Yes

No

Interest in cancer survivors

Low

Moderate

High

Cancer-survivor blame

No

Yes
9.2

9.0

$\begin{array}{ll}7.5 & 1.00\end{array}$

$6.4 \quad 0.99(0.44-2.21)$

$9.5 \quad 1.92(0.78-4.74)$

9.1

10.9

14.0

12.4

9.7

7.8

11.1

10.5

9.7

6.7

9.2

11.4

8.0

7.6

10.3

7.3

10.2

9.3

7.8

10.0

12.1

11.5

8.2

9.4

8.0

9.8

8.2

12.1

9.0

5.9

7.6

10.6
1.00

$1.49(0.92-2.40)$

$2.11(0.82-5.37)$

$2.76(0.99-7.63)$

4.11 (1.30-10.97)

1.00

$0.82(0.41-1.64)$

0.85 (0.38-1.87)

1.00

$0.90(0.65-1.21)$

$0.81(0.59-1.17)$

$0.59(0.36-0.94)$

1.00

$1.08(0.47-2.46)$

$1.32(0.62-2.80)$

1.00

$1.50(1.02-2.22)$

1.00

$1.19(0.72-1.98)$

$1.09(0.60-1.97)$

1.00

1.35 (0.98-1.87)

1.42 (1.07-1.88)

1.00

$0.58(0.35-0.95)$

1.00

$0.66(0.39-1.11)$

1.00

$0.75(0.51-1.11)$

1.00

$0.68(0.51-0.91)$

$0.36(0.22-0.60)$

1.00

$1.36(1.09-1.67)$

aOR, adjusted odd ratio; CI, confidence interval. a) All variables were adjusted for the logistic regression analyses. 
responsible for their own cancer were more likely to have a negative attitude than those who did not.

A lot of study participants had negative attitudes towards cancer survivors. These results are worse than those from a previous study conducted with the same questionnaire in Korea [11]. In our study, the population was more likely to perceive cancer survivors as physically, emotionally, and socially impaired individuals. The percentage of respondents who believed that cancer survivors deserved to be protected in society increased from $56.6 \%$ in the previous study to $77.2 \%$ in our study. Similarly, the percentage of participants who would avoid marrying people with family members who were cancer survivors also increased, from $48.1 \%$ to $63.2 \%$. In addition, except for one out of eight attitude items, all items showed more negative responses than previous research results. These findings indicate that the number of cancer survivors has increased, due to developing medical technology and improved cancer screening; however, intervention to improve awareness for cancer survivors are still insufficient.

Participants who earned more than US $\$ 7,000$ in monthly household income were less likely to have a negative attitude to cancer survivors than those who earn less than US $\$ 1,499$, and people in their 70s, individuals without a religion, and rural residents were more likely to have a negative attitude towards cancer survivors than their 20s, respondents with a religion, and those who live in capital city. Our results regarding the effects of age, income, and city versus rural areas are consistent with previous studies that found a higher frequency of negative perceptions about cancer survivors returning to work among people in rural or urban areas than in metropolitan areas $[11,17]$. Because rural areas are industrial communities that depend more on labor than metropolitan areas, it may be difficult for physically weakened cancer survivors to play traditional roles in rural areas. In addition, because health care in rural areas can be poor quality and it is difficult for local residents to access, it may be difficult for cancer survivors to receive hospital treatment [18]. In fact, cancer survivors in rural areas are more likely to report poor health, psychological distress, and non-cancer comorbidities [19]. These factors may contribute to rural residents having more negative attitudes towards cancer survivors than metropolitan area residents.

Nonsmokers were less likely to have negative attitudes towards cancer survivors. Some studies have found that happiness is related to non-smoking [20]. People with healthy lifestyles are more likely to have higher life satisfaction, whereas people who were exposed to smoking reported unhappiness [20-22]. It is possible that those who do not smoke have a more positive attitude towards life and thus have less negative perceptions of cancer survivors.

People with interest in cancer survivors were less likely to have a negative attitude towards them. Interest, as part of the affective dimensions, has a strong influence on learning and is often associated with positive emotions and curiosity [23]. Interest about cancer positively correlates with all subscales of intentions of proactive behavior towards cancer [24]. In this context, the more a person is interested in cancer survivors, the more they listen to the information about cancer survivors. Through these communications, they become more aware of cancer survivors. Therefore, to reduce the negative perception of cancer survivors shown in this study, we recommend appropriate education and publicity to raise public interest in cancer survivors. One approach could be to use strategic education and messaging to communicate the negative feelings of cancer survivors and then discuss the sources of these emotions [24].

People who blame cancer survivors for their cancer were more likely to have a negative attitude towards cancer survivors than those who do not. Several studies have shown that respondents who attribute some blame to someone with cancer were discriminatory and held stereotyped views $[6,25]$. Knowledge of cancer prevention was associated with blame attribution. People who knew about the connection between lifestyle factors and cancer were more likely to blame cancer survivors for their medical conditions, which resulted in greater negative perceptions towards survivors [6]. Because many public health campaigns focus on raising awareness about preventable causes of cancer, these campaigns might have increased stigma towards cancer survivors [5]. For example, some anti-tobacco campaigns increased stigma and caused negative self-appraisal and feelings of guilt, regret, and perceived blame in people with cancer. Therefore, it can be argued that the focus of campaigns should be shifted from patient blame to more macroscopic industrial problems [5].

This study had several limitations. Respondents may have reported a more positive attitude towards cancer survivors because of social desirability, rather than as a true reflection of their attitude. This would result in an underestimation of the negative attitude towards cancer survivors. In addition, this study focuses on survivors of all cancer types; thus, it is difficult to rule out any differences in attitudes towards different cancer types. The reaction to someone with cancer may depend on the ability to control or prevent the disease. Cancers caused by modifiable lifestyle factors may cause more negative reactions than other types. Therefore, future studies should explore public attitudes towards cancer survivors according to different cancer types.

In summary, we identified negative attitudes towards cancer survivors among Korean adults and characterized some factors related to negative feelings towards cancer survivors. Strategic messaging in education, publicity, and advocacy are required to raise awareness and increase interest in can- 
cer survivors. These activities should highlight social and institutional aspects of cancer, and not transfer the responsibility for disease to cancer patients. Researchers, health care professionals, and communication specialists should work together to build social environments that help cancer survivors get the best treatment and care.

\section{Conflicts of Interest}

Conflict of interest relevant to this article was not reported.

\section{Acknowledgments}

This study was supported by a grant from the National Cancer Center (1610311) and the National R\&D Program for Cancer Control, Ministry of Health and Welfare, Republic of Korea (1731920).

\section{References}

1. Ferlay J, Soerjomataram I, Ervik M, Dikshit R, Eser S, Mathers $\mathrm{C}$, et al. GLOBOCAN 2012 v1.0. Cancer incidence and mortality worldwide: IARC CancerBase No. 11 [Internet]. Lyon: International Agency for Research on Cancer; 2013 [cited 2018 Dec 10]. http://globocan.iarc.fr/.

2. World Health Organization. Cancer prevention and control in the context of an integrated approach: report by the secretariat. Geneva: World Health Organization; 2016.

3. Yoo GJ, Aviv C, Levine EG, Ewing C, Au A. Emotion work: disclosing cancer. Support Care Cancer. 2010;18:205-15.

4. Montazeri A, Tavoli A, Mohagheghi MA, Roshan R, Tavoli Z. Disclosure of cancer diagnosis and quality of life in cancer patients: should it be the same everywhere? BMC Cancer. 2009;9:39.

5. Riley KE, Ulrich MR, Hamann HA, Ostroff JS. Decreasing smoking but increasing stigma? Anti-tobacco campaigns, public health, and cancer care. AMA J Ethics. 2017;19:475-85.

6. Shepherd MA, Gerend MA. The blame game: cervical cancer, knowledge of its link to human papillomavirus and stigma. Psychol Health. 2013;29:94-109.

7. Phelan SM, Griffin JM, Jackson GL, Zafar SY, Hellerstedt W, Stahre M, et al. Stigma, perceived blame, self-blame, and depressive symptoms in men with colorectal cancer. Psychooncology. 2013;22:65-73.

8. Shen MJ, Hamann HA, Thomas AJ, Ostroff JS. Association between patient-provider communication and lung cancer stigma. Support Care Cancer. 2016;24:2093-9.

9. Williamson TJ, Choi AK, Kim JC, Garon EB, Shapiro JR, Irwin $\mathrm{MR}$, et al. A longitudinal investigation of internalized stigma, constrained disclosure, and quality of life across 12 weeks in lung cancer patients on active oncologic treatment. J Thorac Oncol. 2018;13:1284-93.

10. Jung KW, Won YJ, Kong HJ, Lee ES, Community of Population-Based Regional Cancer Registries. Cancer statistics in Korea: incidence, mortality, survival, and prevalence in 2015. Cancer Res Treat. 2018;50:303-16.

11. Cho J, Smith K, Choi EK, Kim IR, Chang YJ, Park HY, et al. Public attitudes toward cancer and cancer patients: a national survey in Korea. Psychooncology. 2013;22:605-13.
12. Cho J, Choi EK, Kim SY, Shin DW, Cho BL, Kim CH, et al. Association between cancer stigma and depression among cancer survivors: a nationwide survey in Korea. Psychooncology. 2013;22:2372-8.

13. Park JH, Park EC, Park JH, Kim SG, Lee SY. Job loss and re-employment of cancer patients in Korean employees: a nationwide retrospective cohort study. J Clin Oncol. 2008;26: 1302-9.

14. Kye SY, Park K. Suicidal ideation and suicidal attempts among adults with chronic diseases: a cross-sectional study. Compr Psychiatry. 2017;73:160-7.

15. Milbury K, Badr H, Carmack CL. The role of blame in the psychosocial adjustment of couples coping with lung cancer. Ann Behav Med. 2012;44:331-40.

16. Korean Statistical Information Service. Household income and expenditure [Internet]. Daejeon: Statistics Korea; 2017 [cited 2018 Dec 5]. Available from: http:// kosis.kr/statisticsList/statisticsListIndex.do?menuld=M_01_01\&vwcd=MT_ZTITLE\&p armTabId=M_01_01\#SelectStatsBoxDiv / .

17. Shim HY, Shin JY, Kim JH, Kim SY, Yang HK, Park JH. Negative public attitudes towards cancer survivors returning to work: a nationwide survey in Korea. Cancer Res Treat. 2016; 48:815-24.

18. Lee YJ. An study on the inepuality of health care resources distribution affected by regional characteristics. J Crit Soc Welf. 2005;21:49-78.

19. Weaver KE, Geiger AM, Lu L, Case LD. Rural-urban disparities in health status among US cancer survivors. Cancer. 2013;119:1050-7.

20. Shiue I. Self and environmental exposures to drinking, smoking, gambling or video game addiction are associated with adult hypertension, heart and cerebrovascular diseases, allergy, self-rated health and happiness: Japanese General Social Survey, 2010. Int J Cardiol. 2015;181:403-12.

21. Grant N, Wardle J, Steptoe A. The relationship between life satisfaction and health behavior: a cross-cultural analysis of young adults. Int J Behav Med. 2009;16:259-68.

22. Kye SY, Park K. Health-related determinants of happiness in Korean adults. Int J Public Health. 2014;59:731-8. 
23. Krapp A. An educational-psychological conceptualization of interest. Int J Educ Vocat Guid. 2007;7:5-21.

24. Heuckmann B, Asshoff R. German high school students' attitudes and interest in cancer and factors influencing proactive behaviour for cancer prevention. J Cancer Educ. 2014;29:497-
505.

25. Marlow LA, Waller J, Wardle J. Variation in blame attributions across different cancer types. Cancer Epidemiol Biomarkers Prev. 2010;19:1799-805. 